Revista

\title{
Multi-Ensayos
}

Vol. 6, núm. 12

ISSN: 2412-3285

https://multiensayos.unan.edu.ni

DOI: https://doi.org/10.5377/multiensayos.v6i12.10118

\section{Algunas notas sobre autonomía en las universidades de España y Nicaragua desde la perspectiva administrativa del derecho}

\section{Some notes on autonomy in the universities of Spain and Nicaragua from the administrative perspective of law}

\author{
Verónica Fabiola Juárez Henríquez ${ }^{1}$ \\ Iván Eugenio Aguilar Fiallos²
}

Recibido: 26 de junio de 2020. Aceptado: 28 de julio de 2020

\section{RESUMEN}

El servicio público de educación superior es ofertado por el Estado nicaragüense y español a través de instituciones especializadas, estas son las universidades, sean de naturaleza pública o privada. El régimen jurídico y administrativo bajo el cual operan estas instituciones es por medio del modelo descentralizado por servicio o por colaboración. De manera que el objetivo de este pequeño estudio es contrastar el grado de autonomía que tienen las instituciones de educación superior en el régimen de descentralización por servicio o por colaboración que los respectivos marcos jurídicos español y nicaragüense les otorgan a las universidades sean éstas públicas o privadas. Para ello, se aborda de forma sucinta una panorámica doctrinaria sobre lo que es descentralización por servicio y colaboración. Se describe los rasgos particulares de las Universidades públicas y privadas tanto en España como en Nicaragua y, finalmente se realiza una comparación de los niveles de autonomía que presentan las instituciones de educación superior en España y Nicaragua. La Metodología utilizada en la investigación jurídica es teórica documental y el tipo de estudio descriptivo y comparativo del grado de autonomía en el régimen de descentralización por servicio o por colaboración de las universidades públicas y privadas de acuerdo a la legislación universitaria de España y Nicaragua. Por lo que las técnicas de investigación fueron esencialmente documentales (fuentes primarias del conocimiento [Constituciones, leyes, etc.], fuentes bibliográficas específicas relacionados con la descentralización administrativa.

Palabras clave: Educación superior; autonomía universitaria; descentralización por servicio; descentralización por colaboración.

\footnotetext{
1 Docente UNAN-León. Correo electrónico: veronica.juarez@cj.unanleon.edu.ni

2 Docente UNAN-León. Correo electrónico: ivan.aguilar@cj.unanleon.edu.ni (c) 2020 Revista Multi-Ensayos.
} 


\section{ABSTRACT}

The public service of higher education is offered by the Nicaraguan and Spanish State through specialized institutions, these are the universities, be they public or private. The legal and administrative regime under which these institutions operate is through the decentralized model by service or by collaboration. Thus, the objective of this small study is to contrast the degree of autonomy that higher education institutions have in the system of decentralization by service or by collaboration that the respective Spanish and Nicaraguan legal frameworks grant to universities, whether they are public or private. . To do this, a doctrinal overview of what decentralization is for service and collaboration is succinctly addressed. The particular features of public and private universities in both Spain and Nicaragua are described and, finally, a comparison is made of the levels of autonomy that higher education institutions present in Spain and Nicaragua. The Methodology used in legal research is documentary theory and the type of descriptive and comparative study of the degree of autonomy in the system of decentralization by service or by collaboration of public and private universities according to university legislation in Spain and Nicaragua. Therefore, the investigation techniques were essentially documentary (primary sources of knowledge [Constitutions, laws, etc.]), specific bibliographic sources related to administrative decentralization.

Keywords: Higher education; university autonomy; service decentralization; collaboration decentralization.

\section{INTRODUCCIÓN}

El derecho a la educación es un derecho fundamental consagrado en los textos constitucionales de los ordenamientos jurídicos español y nicaragüense. En lo que respecta a la educación superior, si bien es cierto, es una función del Estado, le corresponde su gestión, organización y funcionamiento a las Universidades bajo un régimen de autonomía. En tal sentido, encontramos que en el caso español dicha autonomía está consagrada en el apartado 10 del artículo 27 de su Constitución Política y, en el caso nicaragüense, en el artículo 125 de su carta magna. En ambos casos, como veremos más adelante, una ley de la materia regula los términos de sus respectivas autonomías.

Del estudio comparativo de las legislaciones universitarias de España y Nicaragua, se deduce que las universidades en ambos lados del atlántico actúan bajo un cierto grado de discrecionalidad académica, financiera, administrativa y orgánica pero, el grado de autonomía que tienen las mismas en cada uno de estos campos competenciales dependerá exclusivamente de las disposiciones jurídicas de la materia y de la naturaleza pública o privada de la institución de educación superior, lo que indicará la situación de mínima o máxima descentralización del servicio público prestado.

\section{DESARROLLO}

Descentralizar en términos jurídicos "consiste en confiar la realización de algunas actividades administrativas, a órganos que guardan con la administración central del Estado, una relación que no es la de jerarquía, pero sin que dejen de existir las facultades indispensables para conservar la unidad del Poder" (Escorcia, 2009, p. 297).

Antes de reseñar algunas particularidades distintivas del caso español y nicaragüense, es menester resaltar puntos comunes en ambas legislaciones universitarias, en este sentido, partimos de la norma constitucional de ambos países, pues en dicha norma se consagra la autonomía universitaria en los 
artículos 27 inc. 10 (España) y 125 (Nicaragua). El alcance de ésta autonomía se encuentra desarrollado por leyes, para el caso español es la Ley Orgánica de Universidades (en adelante ley 6/2001) y, para el caso nicaragüense es la Ley de Autonomía de las Instituciones de Educación Superior (en adelante ley 89). De manera que, la educación superior es un servicio público orientado a la formación profesional y ciudadana de los estudiantes universitarios y que el contenido de dicha autonomía consiste en: (Art. 2 Ley 6/2001 y Art. 8 Ley 89)

- Autonomía Académica: Pueden por si misma nombrar y remover el personal docente e investigador por medio de los procedimientos y requisitos que ellas mismas señalen, así como la formación y promoción de los mismos; seleccionar a sus alumnos, mediante las pruebas y condiciones necesarias; elaborar y aprobar sus planes y programas de estudios y de investigación, etc.

- Autonomía Financiera: Implica la elaboración del presupuesto interno y la gestión financiera, sin perjuicio de la rendición de cuenta y fiscalización, por la autoridad estatal correspondiente.

- Autonomía Administrativa: Se dispone en todo cuanto se refiere a la gestión administrativa y al nombramiento del personal administrativo y de servicio correspondiente.

- Autonomía Orgánica: Implica que proceden libremente a integrar sus distintos órganos de gobierno y a elegir sus autoridades, a elaborar sus propios estatutos o normas de régimen interno.

La Autonomía confiere, además, la potestad de: Gozar de personalidad jurídica propia y patrimonio propio; Expedir certificados de estudio; cartas de egresados; constancias, diplomas, títulos y grados académicos y equivalencias de estudios del mismo nivel realizados en otras universidades y centros de Educación Superior, nacional o extranjeros; facultad de reconocer los grados académicos y los títulos y diplomas universitarios otorgados en el extranjero; autorizar el ejercicio profesional; la inviolabilidad de los recintos y locales universitarios. La fuerza pública sólo podrá entrar en ellos con autorización escrita de la autoridad universitaria competente (esto último para el caso nicaragüense).

En lo que respecta a las Universidades privadas, cabe destacar, que los textos constitucionales de estos países dentro de una economía de mercado permiten la apertura de centros privados que oferten el servicio de educación, así lo establece el artículo 27 inc. 6 (España) de que personas físicas y jurídicas tendrán la libertad de crear centros docentes dentro del respeto a los principios constitucionales y el artículo 105 (Nicaragua) que garantiza el derecho de establecer servicios privados en las áreas de salud y educación. Por tanto, estas Universidades privadas gozarán de todas las potestades que sus respectivas legislaciones le permitan incluso del alcance de la autonomía.

Sabemos que al Estado se le ha encomendado la obligación de satisfacer necesidades colectivas de interés general, en virtud de ello la actividad de prestación del servicio público puede ser llevada a cabo de forma directa por el Estado de manera centralizada, descentralizada y por medio de empresas privadas a las que el Estado autoriza mediante una concesión. La descentralización administrativa adopta según la doctrina tres modalidades, a saber:

- Descentralización Territorial.

- Descentralización Funcional o por Servicio.

- Descentralización por Colaboración. 
Dado el objeto de estudio que nos ocupa, abordaremos sucintamente las últimas dos modalidades de descentralización.

De manera que, el Estado en su afán de satisfacer las necesidades de interés general, uno de ellos es el servicio público de educación superior, decide que "conviene desprenderlos de la Administración Central, con el objeto de ponerlos en manos de personas con una preparación técnica que garantice su eficaz funcionamiento, así como para evitar un crecimiento anormal del Poder del Estado. La forma de conseguir ese propósito es conceder independencia al servicio y constituirle un patrimonio que le sirva de base a su autonomía funcional" (Op. cit, p. 345), es esto, precisamente, la Descentralización Funcional o por Servicio. Los elementos esenciales de ella, son: "La existencia de un servicio público de orden técnico; la participación de funcionarios técnicos en la dirección del servicio; un estatuto legal [...] que regule la actuación de los funcionarios encargados de dicho servicio; responsabilidad personal y efectiva de los funcionarios; control estatal, ejercido mediante la legalidad de los actos realizados por el servicio descentralizado". (Ibíd)

Cuando un Estado adquiere mayor injerencia en la esfera privada y se le presentan problemas a los que tiene que encontrar una solución, se requiere entonces de la preparación técnica de la que carecen los funcionarios y empleados públicos de carrera o no, por lo que crear organismos especializados para esto recargaría aún más la tarea y los presupuestos de la Administración estatal, ante esta realidad, se autoriza a las organizaciones privadas para que colaboren con el Estado "haciéndolas participar en el ejercicio de la función administrativa. De ésta manera, viene a ser una de las formas del ejercicio privado de las funciones públicas" (Op. cit, p. 347), esto es la descentralización por colaboración. Sus características esenciales son: "El organismo ha de: ser constituido por individuos particulares; de disponer de un patrimonio privado especial, formado por dinero y bienes que los miembros aportan al objeto de su establecimiento; ejercer funciones de interés general para satisfacer necesidades colectivas; la creación del organismo y su personalidad ha de realizarse con la aprobación de la autoridad estatal correspondiente; regirse por sus propios estatutos; las personas empleadas por el organismo no son funcionarios públicos y su ingreso es un acto privado; colabora con el Estado en la satisfacción de una necesidad colectiva". (Ibíd. p. 348),

De lo anterior, podemos concluir, que la diferencia que existe entre los modelos de descentralización administrativa por servicio y por colaboración, estriba en que el organismo que actúa por servicio es parte integrante de la organización administrativa y además la actividad se realiza en nombre y en interés del Estado, en cambio, el organismo que actúa por colaboración realiza la actividad en nombre de la propia organización privada, pero en interés del Estado, además, no es parte integrante del mismo, lo cual no significa que el Estado no posea facultades sobre este organismo privado, al contrario, las facultades que se reserva el Poder Estatal y que a la vez son elementos fundamentales para clasificar a un organismo privado como colaborador del Estado son: El poder de autorizar a las instituciones admitidas para colaborar en la función del Estado; el poder de retirar la autorización; el poder de vigilancia y control que sean necesarias para mantener esa colaboración dentro de los límites legales, tales como poder vigilar la selección del personal, comprobar de manera continua el funcionamiento de la institución colaboradora (Cfr. Fraga, 1990, p. 349). 


\section{SIMILITUDES Y DIFERENCIAS QUE PRESENTAN LAS NORMAS JURÍDICAS EN EL CASO ESPAÑOL Y NICARAGÜENSE}

A continuación, se detallan algunos aspectos importantes a destacar contenidos en la Ley Orgánica de Universidades (ley 6/2001) del caso español y, para el caso nicaragüense la Ley de Autonomía de las Instituciones de Educación Superior (ley 89). Esto con el objeto de visualizar el grado de discrecionalidad que tienen estas instituciones a la hora de ejercitar todos los componentes de la autonomía universitaria (académica, administrativa, financiera y orgánica). Por ello, nos centramos en los principales órganos colegiados de gobierno (España: Consejo Social, Consejo de Gobierno, Claustro Universitario/Nicaragua: Consejo Universitario) descritos por sus respectivas normas jurídicas. Antes estudiaremos la naturaleza jurídica de las universidades públicas y privadas y conocer cuáles son sus respectivos regímenes jurídicos.

\section{NATURALEZA JURÍDICA DE LAS INSTITUCIONES DE EDUCACIÓN SUPERIOR}

\begin{tabular}{l}
\hline ESPAÑA \\
\hline $\begin{array}{l}\text { - } \\
\text { - }\end{array}$ Créblicas y Privadas: \\
los órganos legislativos: Comunidades \\
Autónomas/las Cortes Generales. (Art. 3 \\
y 4) \\
\hline - A propuestas del Gobierno, y del \\
Consejo de Gobierno de la Comunidad \\
Autónoma. \\
\hline \begin{tabular}{l} 
Requisito previo un informe de \\
la Conferencia General de Política \\
Universitaria. \\
\hline Comunidades Autónomas autorizarán el \\
comienzo de las actividades universitarias
\end{tabular} \\
\hline
\end{tabular}

RÉGIMEN JURÍDICO DE LAS UNIVERSIDADES

\begin{tabular}{|l|l|}
\hline \multicolumn{2}{|l|}{ ESPAÑA } \\
\hline - & Constitución Política \\
\hline - & Ley Orgánica de las universidadeS \\
\hline - & $\begin{array}{l}\text { Normas que dicten el Estado y las Comunidades } \\
\text { Autónomas, en el ejercicio de sus respectivas } \\
\text { competencias. }\end{array}$ \\
\hline - & Ley de su creación (universidades públicas) \\
\hline - & Ley de reconocimiento (universidades privadas) \\
\hline - & $\begin{array}{l}\text { Estatutos, elaborados por ellas mismas. } \\
\text { (Universidades públicas) }\end{array}$ \\
\hline - & $\begin{array}{l}\text { Previo control de legalidad, aprobados por el } \\
\text { Consejo de Gobierno de la Comunidad Autónoma, }\end{array}$ \\
\hline - & $\begin{array}{l}\text { Los Estatutos entrarán en vigor a partir de su } \\
\text { publicación en el Boletín Oficial de la Comunidad }\end{array}$ \\
& $\begin{array}{l}\text { Autónoma./ "Boletín Oficial del Estado". (Art. } 27 \text { Cn } \\
\text { y Art. } 6 \text { Ley 6/2001) }\end{array}$ \\
\hline
\end{tabular}

\begin{tabular}{l}
\hline NICARAGUA \\
\begin{tabular}{|l|l|}
\hline - & Públicas y Privadas: \\
\hline & $\begin{array}{l}\text { Creadas mediante Ley en la que se les } \\
\text { otorga Personalidad Jurídica por la } \\
\text { Asamblea Nacional. }\end{array}$ \\
\hline - & Requisito previo: Autorización del \\
& Consejo Nacionalde Universidades (CNU). \\
\hline
\end{tabular}
\end{tabular}

\section{NICARAGUA}

- Tanto Públicas como Privadas

- Constitución Política.

- Ley. 89 "Ley de Autonomía"

- Ley. 147 "Ley general de Personas Jurídicas Sin Fines de Lucro"

- Leyes de la República.

- Estatutos o Reglamentos aprobados por las Universidades.

- Resoluciones dictadas por el CNU. 


\section{ÓRGANOS DE GOBIERNO DE LAS UNIVERSIDADES PÚBLICAS Y PRIVADAS}

\section{ESPAÑA}

- Colegiados: Consejo Social, Consejo de Gobierno, Claustro Universitario, Juntas de Escuela y Facultad y Consejos de Departamento.

- Unipersonales: el Rector (a), Vicerrectores (as), Secretario (a) General, Gerente, Decanos (as) de Facultades, Directores (as) de Escuelas, de Departamentos y de Institutos Universitarios de Investigación. (Arts: 13, 14, 15, 16, 18-26, 27, 81, 82 Ley 6/2001)

\section{CONSEJO SOCIAL (C.S.):}

\section{ESPAÑA}

- Órgano de participación de la sociedad en la universidad;

- Composición:

\section{Comunidad Autónoma (CA):}

- Personalidades de la vida cultural, profesional, económica, laboral y social/ EI Presidente C.S. es nombrado por la CA

\section{Comunidad Universitaria:}

- Rector, Secretario General y el Gerente.

- Consejo de Gobierno: un profesor, un estudiante y un representante del personal de administración y servicios.

\section{NICARAGUA}

- Colegiados: El Consejo Universitario; El Consejo de Facultad; El Consejo de Dirección de Escuela

- Unipersonales: El Rector; EI Decano de Facultad; y el Director de Escuela, donde los hubiese.

\section{- Supervisan las actividades} de carácter económico de la universidad y del rendimiento de sus servicios.

- Promover la colaboración de la sociedad en la financiación de la universidad.

- Aprueba la propuesta de Presupuesto del Consejo de Gobierno.

\section{NICARAGUA}

- Este órgano de participación social no existe. 


\section{ÓRGANOS DE GOBIERNO DE LAS UNIVERSIDADES PÚBLICAS Y PRIVADAS}

\section{ESPAÑA}

\section{Consejo Social:}

- Supervisa las actividades de carácter económico /rendimiento de sus servicios.

- Aprueba la propuesta de Presupuesto del Consejo de Gobierno.

\begin{tabular}{l} 
Claustro Universitario: \\
\begin{tabular}{|l|l|} 
- máximo órgano de representación de la \\
comunidad universitaria.
\end{tabular} \\
\hline - $\begin{array}{l}\text { Formado: Rector, Secretario General; Gerente, } \\
\text { (Composición según los Estatutos) }\end{array}$ \\
\hline - Elabora los Estatutos; \\
\hline - Elección del Rector (según sea el caso) \\
\hline
\end{tabular}

\section{NICARAGUA}

\section{CONSEJO UNIVERSITARIO:}

- Máximo órgano de gobierno de la Universidad;

- Dictar sus propios reglamentos internos y aprobar los Estatutos y los diferentes reglamentos;

- Nombrar, a propuesta del Rector, los vice- rectores y el Secretario General de la Universidad;

- Aprobar el Presupuesto General de gastos e ingresos de la Universidad y los planes prospectivos de la institución y las facultades;

- Aprobar la creación, modificación o supresión de carreras, previo dictamen del Consejo Nacional de Universidades;

- Normarygarantizarlas elecciones universitarias;

- Aprobar los nombramientos y remociones del personal.
CONSEJO DE GOBIERNO:

- Órgano de gobierno de la Universidad.

- Establece las líneas estratégicas, programáticas de la Universidad, y directrices para su aplicación (enseñanzas, investigación, recursos humanos y económicos)

- Elaboran los presupuestos.

- Composición:

- Rector; Secretario General; Gerente,

- Vicerrectores, Decanos y Directores,

- Hasta un máximo de 3 miembros del Consejo Social, no pertenecientes a la comunidad universitaria. (Estatutos)

- Un máximo de 50 miembros (representación de la comunidad universitaria) 
En estas tablas comparativas se puede apreciar que las competencias de: elaboración de presupuestos; aprobación del presupuesto; supervisión económica y de rendimiento; elaboración de los Estatutos y el sistema de elección del Rector, son todas ellas competencias repartidas entre todos estos órganos colegiados, en cambio, para el caso nicaragüense, todas estas competencias recaen en un solo órgano el Consejo Universitario que es el máximo órgano de gobierno de las universidades nicaragüenses, el cual está integrado exclusivamente por miembros de la comunidad universitaria. Nótese que, en materia de autonomía financiera, para el caso español, las atribuciones económicas y presupuestarias son elaboradas por los Consejos de Gobierno de las universidades, pero la aprobación de la política presupuestaria depende de otro órgano colegiado, el Consejo Social, que está integrado, además de representantes de la comunidad universitaria, por personas de distintos sectores sociales, designados, a igual que el Presidente de este órgano, por los gobiernos autonómicos de cada una de las Comunidades Autónomas de España en donde hay presencia de universidades. De tal manera que el grado de autonomía financiera es menor con respecto al caso nicaragüense cuyo Consejo Universitario goza de mayor discrecionalidad a la hora de elaboración, aprobación, ejecución y revisión de cuentas.

\section{ORGANISMOS QUE COORDINAN, COOPERAN Y COLABORAN CON LAS UNIVERSIDADES}

\section{ESPAÑA}

- Conferencia General de Política Universitaria. (Art. 27-31 Ley 6/2001)

- Órgano de concertación, coordinación y cooperación de la política general universitaria / articular sus políticas al espacio europeo de educación superior y su interrelación con las políticas de investigación científica y tecnológica.

- Elabora informes sobre la programación general de la enseñanza universitaria que son determinantes en la creación y reconocimientos de Universidades.

- Aprobar los criterios de coordinación sobre las actividades de evaluación, certificación y acreditación.

- Ministro de Educación Cultura y Deporte y responsables de educación universitaria en las C.A.

\begin{tabular}{|c|c|}
\hline NIC & EARAGUA \\
\hline & $\begin{array}{l}\text { Consejo } \quad \text { Nacional } \\
\text { Universidades (CNU) }\end{array}$ \\
\hline & $\begin{array}{l}\text { Ministro de Educación puede } \\
\text { participar en las reuniones de } \\
\text { Rectores en calidad de invitado. } \\
\text { Sólo voz pero No voto. }\end{array}$ \\
\hline & $\begin{array}{l}\text { Elaborar y coordinar la política } \\
\text { nacional de la Educación } \\
\text { Superior del país, }\end{array}$ \\
\hline & $\begin{array}{l}\text { Proponer la política de } \\
\text { distribución de los fondos } \\
\text { asignados a las universidades; }\end{array}$ \\
\hline & $\begin{array}{l}\text { Dictaminar sobre la apertura o } \\
\text { cierre de carreras; }\end{array}$ \\
\hline & $\begin{array}{l}\text { Autorizar la creación de nuevas } \\
\text { universidades. }\end{array}$ \\
\hline & Órganos Consultivos internos: \\
\hline$\bullet$ & Asamblea General Universitaria \\
\hline & Asamblea General de Facultad \\
\hline
\end{tabular}




\section{NICARAGUA}

\section{CONSEJO UNIVERSITARIO:}

- Máximo órgano de gobierno de la Universidad;

- Dictar sus propios reglamentos internos y aprobar los Estatutos y los diferentes reglamentos;

- Nombrar, a propuesta del Rector, los vice- rectores y el Secretario General de la Universidad;

- Aprobar el Presupuesto General de gastos e ingresos de la Universidad y los planes prospectivos de la institución y las facultades;

- Aprobar la creación, modificación o supresión de carreras, previo dictamen del Consejo Nacional de Universidades;

- Normarygarantizar las elecciones universitarias;

- Aprobar los nombramientos y remociones del personal.
Como se puede observar, la política nacional sobre materia de educación superior, en el caso nicaragüense es trazada exclusivamente por el CNU y, aunque el Ministro de Educación puede participar en las reuniones del CNU, éste no tiene poder de decisión por medio de su voto, sino que sólo cuenta con la facultad de ser escuchado. Mientras que, en el contexto español, el titular del Ejecutivo a través del Ministro de Educación y el Ministro competente en materia de universidades, cuenta con poder de decisión ya que poseen las facultades de voz y voto dentro de la Conferencia General de Política Universitaria y el Consejo de Universidades. Por tanto, la autonomía académica en las universidades españolas es más limitada con respecto a las universidades nicaragüenses.

\section{DEL RÉGIMEN ECONÓMICO Y FINANCIERO DE LAS UNIVERSIDADES PÚBLICAS}

\begin{tabular}{|c|c|}
\hline \multicolumn{2}{|c|}{ ESPAÑA } \\
\hline & $\begin{array}{l}\text { Transferencias para gastos corrientes y } \\
\text { de capital fijadas, anualmente, por las } \\
\text { Comunidades Autónomas. }\end{array}$ \\
\hline & Gestión de ingresos propios \\
\hline & $\begin{array}{l}\text { Beneficios fiscales: Universidades públicas } \\
\text { y privadas (Ley 30/1994, de Fundaciones e } \\
\text { Incentivos Fiscales a la Participación Privada en } \\
\text { Actividades de Interés General) }\end{array}$ \\
\hline \multicolumn{2}{|c|}{ Comunidades Autónomas: } \\
\hline & $\begin{array}{l}\text { Control de las inversiones, gastos e ingreso } \\
\text { aquéllas, mediante técnicas de auditoría, } \\
\text { la supervisión de los Consejos Sociales. (Art } \\
80,81 \text { Ley } 6 / 2001 .)\end{array}$ \\
\hline
\end{tabular}

\section{NICARAGUA}

- Estarán exentos de toda clase de impuestos y contribuciones fiscales, regionales y municipales.

- Sus bienes y rentas no podrán ser objeto de intervención, expropiación ni embargo, excepto cuando la obligación que se haga valer, tenga su origen en contratos civiles, mercantiles o laborales.

- Recibirán una aportación anual del 6\% del Presupuesto General de la República, la cual se distribuirá de acuerdo con la ley.

- El Estado podrá otorgar aportaciones adicionales para gastos extraordinarios.

- Gestiona sus ingresos propios

- Rendición de cuentas/Contraloría General de la República. 


\section{ASPECTOS PARTICULARES DEL CASO NICARAGÜENSE}

Cabe mencionar que las Universidades señaladas en lo preceptuado por la ley 89 y que forman parte del Consejo Nacional de Universidades (CNU), reciben el financiamiento del Estado correspondiente al $6 \%$ de los ingresos ordinarios y extraordinarios establecidos en el Presupuesto General de la República para el año correspondiente, independientemente del origen de dichos ingresos y que el CNU administra y lo distribuye a las universidades que lo conforman. (Art. 1, Ley 151; Art. 55 inc. 1, Ley 89)

De acuerdo a la Ley 89, las Instituciones de educación superior correspondiente a las Universidades Públicas, son únicamente cuatro: Universidad Nacional Autónoma de Nicaragua, León (UNAN-León); Universidad Nacional Autónoma de Nicaragua, Managua (UNAN-Managua); Universidad Nacional de Ingeniería (UNI); y la Universidad Nacional Agraria (UNA). Las únicas universidades privadas que gozan del financiamiento estatal del $6 \%$ del presupuesto general de la República: Universidad Centroamericana (UCA); la Universidad Politécnica de Nicaragua (UPOLI). En 1996 mediante Ley N²18, ingresaron también al CNU dos universidades comunitarias de la Costa Caribe: la Bluefields Indian and Caribbean University (BICU) y la Universidad de las Regiones Autónomas de la Costa Caribe de Nicaragua (URACCAN). Y, por último, Universidades Regionales de orden religiosa católica: Universidad Católica Agropecuaria del Trópico Seco (UCATSE) y Universidad Internacional de Agricultura y Ganadería de Rivas (UNIAG).

Sin embargo, este modelo de descentralización por servicio llevado a cabo por las Universidades públicas en nombre y en interés del Estado, conviven con el modelo de descentralización por colaboración, cuya actividad es realizada en nombre de la propia organización privada, colaborando únicamente con el Estado, pero sujeto a su vigilancia y control dentro de los límites legales, éstas son por supuesto las Universidades privadas, las cuales se proliferaron a partir de los años noventa, pues el clima jurídico y político del país favoreció enormemente al desarrollo de la iniciativa privada. Con respecto a la educación, la Constitución en su artículo 123 autoriza que los centros privados dedicados a la enseñanza pueden funcionar en todos los niveles. Es así, que abrieron sus puertas 43 universidades privadas, de manera que, actualmente funcionan en Nicaragua 53 Instituciones de Educación Superior entre las que figuran las relacionadas en la Ley 89 y las privadas (Tunnermann, 2007).

\section{CONCLUSIONES}

Del estudio de las legislaciones universitarias de España y Nicaragua, llegamos a una serie de conclusiones acerca del grado de autonomía que tienen las Universidades públicas de ambos países, pues en el caso de las Universidades privadas, éstas sólo están sometidas a controles de autorización y vigilancia de parte de la Administración pública en aspectos puntuales para el buen funcionamiento de la prestación del servicio, teniendo éstas un grado de autonomía mayor con respecto a las universidades públicas, pues es claro que no reciben fondos públicos. De manera que, analizando el contenido de la Autonomía para las Universidades Públicas de ambos países, tenemos:

Autonomía Académica: Las Universidades desarrollan sus propias atribuciones académicas con cierto grado de discrecionalidad, en el caso español, el nivel de autonomía es bajo debido a que la política general de educación es trazada en la Conferencia General de Política Universitaria conformada por 
la autoridad central y de las Comunidades Autonómicas y, con un nivel máximo de discrecionalidad en el caso nicaragüense pues su política nacional de educación universitaria la determina el CNU que está integrado exclusivamente por Rectores con participación del Ministro de Educación, pero como colaborador sin decisión alguna, pues no tiene la facultad de votar, es como puente de comunicación de la autoridad central con las universidades.

Autonomía Financiera: Las Universidades públicas de España elaboran sus presupuestos (Consejo de gobierno) pero su aprobación depende del Consejo Social cuyo Presidente del mismo, es electo por la Comunidad Autónoma, por tanto, tiene un nivel limitado de autonomía financiera no por la razón de que sea éste órgano quien fiscalice la ejecución del presupuesto ya que esto es una manera de control de las Comunidades Autónomas, sin perjuicio del control que realicen los órganos de cuentas de éstas Comunidades y del Tribunal de Cuentas de España, sino que es limitada su autonomía porque la Universidad puede elaborar su presupuesto pero aprobárselo corresponde a un órgano mixto (composición: Social (C.A)/Comunidad Universitaria) que guarda relación directa con la Comunidad Autónoma.

En el caso nicaragüense, dicha autonomía es máxima porque las Universidades que se benefician con el $6 \%$ del presupuesto general de la República, captan estos fondos públicos y proceden a elaborar sus presupuestos de gastos e ingresos, los aprueban y los ejecutan, debiendo rendir cuentas a la Contraloría General de la República.

Autonomía Orgánica: En ambos países las Instituciones de Educación superior establecen las formas de autogobierno y de auto-legislación, sin embargo, los niveles de discrecionalidad que adoptan las autoridades en sus campos de acción son limitados en el caso español por las razones antes señalas $y$, además, porque en el caso de aprobación de sus Estatutos tienen que pasar por un control político de las Comunidades Autónomas para poder ser aprobados y entrar en vigencia a partir de su publicación en los Boletines Oficiales autonómicos y estatal, en cambio, en las Universidades nicaragüenses no se necesita de ese control de legalidad por parte del ente municipal o estatal, mucho menos sea necesario la publicación del mismo en el Diario Oficial del Estado.

\section{FUENTES DE CONOCIMIENTO LEGALES:}

- Las Cortes (1978, 27 de diciembre). Constitución Española de 1978. Boletín Oficial del Estado, $n^{\circ} .311$. Con Reformas Parciales (1992, 28 de agosto) BOE, n. 207. Y (2011, 27 de septiembre) BOE, nº 233.

- Asamblea Nacional Constituyente de Nicaragua (1987, 9 de enero). Constitución Política de Nicaragua de 1987. La Gaceta Diario Oficial No. 5. Con sus reformas de 1990, 1995, 2000, 2004, 2005, 2007 y 2014.

- Las Cortes Generales (2001, 21 de diciembre). Ley Orgánica 6/2001, de 21 de diciembre, de Universidades. Boletín Oficial del Estado, n. 307. Referencia: BOE-A-2001-24515

- La Asamblea Nacional (1990, 5 de abril). Ley No. 89 Ley de Autonomía de las Instituciones de Educación Superior. La Gaceta Diario Oficial No. 77.

- La Asamblea Nacional (1992, 9 de septiembre). Ley N 151, Ley de Interpretación Auténtica del arto. 55, inciso 1 de la ley de las Instituciones de Educación Superior de Nicaragua. La Barricada. 
- La Asamblea Nacional (1996, 5 de septiembre). Ley No. 218, Ley para la Asignación del Presupuesto Universitario e Inclusión de las Universidades BICU y URACCAN en la Ley de Autotomía de las Instituciones de Educación Superior. El Nuevo Diario.

\section{FUENTES DOCTRINARIAS:}

- Fraga, G. (1990). Derecho Administrativo. México: Porrúa S.A.

- Escorcia, F. (2009). Derecho Administrativo (Primera Parte). Managua: Editorial Jurídica.

- Tunnermann, C., Lopez-Segrera, F. \& Weise, C. (2007, 19 al 21 de julio). Educación Superior: Relevancia Social y Utilidad de la Educación Superior en Cuba, Nicaragua y Bolivia: Ejemplos de Buenas Prácticas. (ponencia) Foro UNESCO, Trinidad y Tobago. 